BULLETIN OF THE

AMERICAN MATHEMATICAL SOCIETY

Volume 77, Number 6, November 1971

\title{
REPRESENTATION THEORY FOR DIVISION ALGEBRAS OVER LOCAL FIELDS (TAMELY RAMIFIED CASE)
}

\author{
BY ROGER HOWE
}

Communicated by Calvin C. Moore, April 21, 1971

Aside from intrinsic interest there are three (related) reasons for studying the unitary representations of $p$-adic division algebras.

(1) They provide heuristics for more difficult (e.g., noncompact) $p$-adic groups.

(2) They would be basic building blocks in a theory of representations of reductive algebraic $p$-adic groups based on the philosophy of cusp forms.

(3) There seem to be deep relations of representations of division algebras with representations of $\mathrm{Gl}_{n}$, having implications in the theory of automorphic forms. This was pointed up in JacquetLanglands [2].

We announce here, for the tamely ramified case, a classification of the representations (Theorem 1), and a result related to (3) (Theorem 2). I would like to thank R. P. Langlands for some stimulating conversations, and in particular for telling me of the likelihood of Theorem 2.

Let $F$ be a locally compact non-archimedean field of residual characteristic $p$. Let $R$ be its maximal order, $\pi$ a prime element, $F \times$ its multiplicative group, and $U=1+\pi R \subseteq F^{\times}$. Let $D$ be a division algebra over $F$. Let $S, \Pi, D^{\times}, V$ be its maximal order, and so forth. We will say $D$ is tamely ramified if its degree, $n$, is prime to $p$. This is the same as to say all its commutative subfields are tamely ramified over $F$.

Let $F^{\prime}$ be a finite extension, with maximal order $R^{\prime}$, prime $\pi^{\prime}$, $U^{\prime}=1+\pi^{\prime} R^{\prime}$ and multiplicative group $F^{\prime \times}$. Let $N\left(F^{\prime} / F\right): F^{\prime \times} \rightarrow F^{\times}$ be the norm map. Let $\psi$ be a character of $F^{\prime \times}$ and $A \subseteq F^{\prime \times}$ a subgroup. We will say $\psi$ is nondegenerate on $A$ if there is no proper subextension $F^{\prime \prime}, F \subseteq F^{\prime \prime} \subset F^{\prime}$, such that ker $N\left(F^{\prime} / F^{\prime \prime}\right) \cap A \subseteq$ ker $\psi \cap A$. Suppose now $F^{\prime}$ is tamely ramified over $F$. We will say a character $\psi$ of $F^{\prime x}$ is admissible if

(a) $\psi$ is nondegenerate on $F^{\prime \times}$, and

(b) if on $U^{\prime}, \psi=\psi^{\prime \prime} \circ N\left(F^{\prime} / F^{\prime \prime}\right)$, where $\psi^{\prime \prime}$ is nondegenerate on $U^{\prime \prime} \subseteq F^{\prime \prime} \times$, then $F^{\prime}$ is unramified over $F^{\prime \prime}$.

AMS 1970 subject classifications. Primary 22D10, 22E50

Copyright (c) American Mathematical Society 1971 
In particular, $\psi$ is admissible if it is nondegenerate on $U^{\prime}$.

For a locally compact group $G$, let $\hat{G}$ be the set of equivalence classes of irreducible representations. Let $\hat{F}^{\circ} \times$ be the set of admissible characters of $\hat{F}^{\prime} \times$.

THEOREM 1. Let $D$ be a tamely ramified division algebra of odd degree over $F$, and let $p$ also be odd.

(a) For any subfield $F^{\prime} \subseteq D$, there is a finite-to-one map $\alpha: \hat{F}^{\circ} \times \rightarrow \hat{D}^{\times}$.

(b) If $F_{1}^{\prime}, F_{2}^{\prime}$ are conjugate in $D^{\times}$, then this conjugacy induces a map $c: F_{1}^{\prime} \times \leftrightarrow F_{2}^{\prime} \times$. The triangle

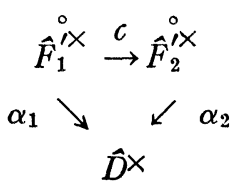

commutes. In particular $\alpha_{1}\left(\hat{F}_{1}^{i} \times\right)=\alpha_{2}\left(\hat{F}_{2}^{i} \times\right)$. If $F_{1}^{\prime}, F_{2}^{\prime}$ are not conjugate, then the images of $\alpha_{1}$ and $\alpha_{2}$ are disjoint.

(c) $\hat{D}^{\times}$is the union of the images of the $\alpha$ 's.

REMARKs. (a) Colloquially we may say $\hat{D}^{\times}$is parametrized by admissible characters of subfields of $D . \alpha: \hat{F}^{\times} \rightarrow \hat{D}^{\times}$is the dual map to $N(D / F), D^{\times} \rightarrow F^{\times}$, the reduced norm map.

(b) There is an analogous theorem for $V$, which in many ways is nicer, but is more difficult to state.

(c) The restriction to odd $n$ and odd $p$ is not serious. In fact, the theorem holds as stated for any $n, p$ relatively prime. However, owing to certain computational difficulties, the indicated parametrization is less satisfactory in those cases. The parametrization in the theorem has a certain degree of naturality, which doubtless is true in the other cases, but which I have not verified.

(d) There are strong analogies between this result and the CartanWeyl highest weight theory.

(e) The proof is based on an analysis of the adjoint action of $D$ on itself, and is based on the Kirillov picture for compact $p$-adic groups, as outlined in [1].

(f) The considerations of the proof also give "most" of the representations of $D$ when $n$ is divisible by $p$, and all the representations when $n=p[1]$. In characteristic zero, the Kirillov picture is also available. However, the precise details for general $n$ will require much more work than the tamely ramified case. Even for $n=p$, the "correct" parametrization is unclear. 
(g) Larry Corwin has independently arrived at a version of Theorem 1. [4].

(h) Our results to some extent parallel those obtained by Shintani

Now let Ad denote the adjoint action of $D^{\times}$on itself by inner automorphisms so that $D^{\times} / \operatorname{Ad} D^{\times}$is the space of conjugacy classes of $D^{\times}$.

Let $K$ be the compositum of all extensions of degree $n$ of $F . K$ is a finite extension of $F$ (see Weil [3]). Let $\operatorname{Gal}(K / F)$ be the galois group of $K$ over $F$. It is known [3] that there is a natural map

$$
\sigma: D / \operatorname{Ad} D^{\times} \rightarrow K / \operatorname{Gal}(K / F)
$$

defined as follows. If $x \in D, x$ generates a certain extension $F^{\prime}$ of $F$ of degree dividing $n$. Thus there is an injection $i: F^{\prime} \rightarrow K$. Then the image of $i(x)$ in $K / \operatorname{Gal}(K / F)$ is $\sigma(x) . \sigma$ is a homeomorphism onto its image. It is even, in a certain sense, an isometry.

It is known [3] that $D$ contains all extensions of $F$ of degree $n$. Thus, if $D_{1}, D_{2}$ are two division algebras of degree $n, \sigma\left(D_{1}\right)=\sigma\left(D_{2}\right)$. Thus $\sigma_{2}^{-1} \circ \sigma_{1}$ defines a homeomorphism

$$
\theta_{12}: D_{1}^{\times} / \operatorname{Ad} D_{1}^{\times} \rightarrow D_{2}^{\times} / \operatorname{Ad} D_{2}^{\times} \text {. }
$$

Let $C_{0}^{\infty}\left(D_{i}^{\times} /\right.$Ad $\left.D_{i}^{\times}\right)=\mathfrak{a}_{i}$ be the space of locally constant class functions of compact support on $D . \mathfrak{a}_{i}$ is an algebra in two ways; by pointwise multiplication and by convolution. By fourier transform $\mathfrak{a}_{i}$ may be viewed as an algebra of functions on $\hat{D}_{i}^{\times}$, and convolution transforms into pointwise multiplication on $\hat{D}_{i}^{\times}$. Clearly $\theta_{12}^{*}: \mathfrak{a}_{2} \rightarrow \mathfrak{a}_{1}$ is an algebra isomorphism for the pointwise multiplication (on $D_{i}^{\times} / \operatorname{Ad} D_{i}^{\times}$).

Theorem 2. Again take $n$ odd and prime to $p$, and $p$ odd. Then $\theta_{12}^{*}: a_{2} \rightarrow \mathfrak{a}_{1}$ is also an isomorphism for the algebra structures given by convolution. In other words, there is a natural homeomorphism $\Phi_{12}: \hat{D}_{1}^{\times}$ $\rightarrow \hat{D}_{2}^{\times}$, dual to $\theta_{12}$, which via fourier transform, also induces $\theta_{12}^{*}$. In other words, there is a bijection $\Phi_{12}: \hat{D}_{1}^{\times} \rightarrow \hat{D}_{2}^{\times}$such that if $U \in \hat{D}_{1}^{\times}$, and $\xi(U)$ is its character, then $\xi(U)=\theta_{12}^{*}\left(\xi\left(\Phi_{12}(U)\right)\right)$. $\Phi_{12}$ commutes with the parametrization given in Theorem 1.

Remarks. (a) Again, this should hold for all $n$. The restriction to odd $n$ could be removed without a major new effort, I believe, but the restriction of $n$ prime to $p$ presents large technical difficulties. Even in the case $n=p$, where one knows the representations, there 
are structural complications obstructing the computation of characters.

(b) The proof is very computational, long and tedious, and will hopefully be improved upon. It proceeds by developing an algorithm for computing characters. The algorithm is then shown to be independent of division algebra. An explicit character formula is not developed. Such a formula, however, would be a very desirable thing to have. The proof also uses a very detailed analysis of the structure of a tamely ramified division algebra.

(c) It is not hard to see that Theorem 2 is "approximately true" by embedding $D_{1}^{\times}$and $D_{2}^{\times}$into a suitable larger group, for example $\mathrm{Gl}_{n}\left(F_{n}\right)$, where $F_{n}$ is the unramified extension of $F$ of degree $n$.

Further progress by Larry Corwin and myself permits us to compute characters explicitly. The results are very suggestive, and, particularly in the unramified case, are reminiscent of Weyl's character formula. Details will appear in a joint paper.

\section{REFERENCES}

1. R. Howe, Kirillov theory for compact P-adic groups (in preparation).

2. H. Jacquet and R. P. Langlands, Automorphic forms on $\mathrm{Gl}_{2}$, Lecture Notes in Math., no. 114, Springer-Verlag, Berlin and New York, 1970.

3. A. Weil, Basic number theory, Die Grundlehren der math. Wissenschaften, Band 144, Springer-Verlag, Berlin and New York, 1967. MR 38 \#3244.

4. T. Shintani, On certain square-integrable irreducible unitary representations of some p-adic linear groups, J. Math. Soc. Japan 20 (1968), 522-565.

State University of New York, Stony Brook, New York 11790

Current address: Institute for Advanced Study, Princeton, New Jersey 08540 